\title{
Stage 1 Palliation of Hypoplastic Left Heart Syndrome: What the Pediatric Anesthesiologist Needs to Know
}

\author{
Dawn M. Sweeney ${ }^{1} \cdot$ Jane $^{\text {Arcadi }^{1}}$ \\ Published online: 9 May 2019 \\ (C) Springer Science+Business Media, LLC, part of Springer Nature 2019
}

\begin{abstract}
Purpose of Review The purpose of this review is to discuss the features of stage 1 palliation of hypoplastic left heart syndrome (HLHS). Comparison will be made between traditional surgical approaches and hybrid approaches. Anesthetic considerations for infants with these types of palliations will be discussed.

Recent Findings While historically surgical palliation in the neonatal period or neonatal heart transplant has been the primary means of survival for neonates with HLHS, in the last two decades, more centers have been performing hybrid palliation of this defect. This palliation can be performed with the expectation that a comprehensive second-stage palliation will be done in the next several months, or it can be used as a bridge to transplant. Other uses are also being explored as research continues.

Summary Advances in the care of neonates with HLHS have led to increased survival. As surgical technique and non-surgical care of these infants continue to improve, the pediatric anesthesiologist will need to be familiar with the anatomic and physiologic implications of the various types of palliation.
\end{abstract}

Keywords Hypoplastic left heart syndrome $\cdot$ Anesthesia $\cdot$ Norwood procedure $\cdot$ Sano procedure $\cdot$ Hybrid palliation $\cdot$ Norwood procedure $\cdot$ Sano modification

\section{Introduction}

Prior to 1980 when William Norwood, MD, and colleagues published a paper describing their experience with providing palliative surgery for infants with aortic atresia, a diagnosis of hypoplastic left heart syndrome was fatal [1]. Neonatal heart transplants were not performed at that time, primarily due to a lack of infant donors. After the transplant of a baboon heart into a neonate with HLHS in 1984, heart transplants for neonates became more common [2]. In 1983, Norwood and his colleagues published their experience with a multistage approach to palliation of HLHS [3]. While the "Norwood"

This article is part of the Topical Collection on Pediatric Anesthesia

Dawn M. Sweeney

Dawn_sweeney@urmc.rochester.edu

Jane Arcadi

Jane_arcadi@urmc.rochester.edu

1 Department of Anesthesiology and Perioperative Medicine, University of Rochester Medical Center, 601 Elmwood Ave, Box 604, Rochester, NY 14642, USA procedure remains the mainstay of surgical palliation for HLHS, the postoperative care of these patients is complicated by the need for extremely careful control of both systemic and pulmonary vascular resistance.

As mortality rates in the 1990 s could reach 40 to $50 \%$ depending on the center, surgeons and scientists continued to explore options to ensure adequate pulmonary blood flow using techniques other than the modified Blalock-Taussig shunt as described by Norwood. In 2003, Shunji Sano, MD, and his colleagues published an article delineating their experience using a right ventricle to pulmonary artery shunt for first-stage palliation of HLHS [4]. The primary benefit of the "Sano" modification was the decreased need for manipulation of the pulmonary vascular resistance in the immediate postoperative period.

While the Sano modification made postoperative care of neonates after palliation of HLHS somewhat less complicated, the procedure still required a period of deep hypothermic circulatory arrest and a significant amount of time on cardiopulmonary bypass. Concerns that the circulatory arrest period and cardiopulmonary bypass were contributing to negative neurologic outcome led investigators to continue research into alternative methods of palliation. 
As cardiac catheterization techniques became more sophisticated and smaller equipment became available, centers began stenting the ductus arteriosus for various lesions including HLHS. In 1993, Gibbs et al. reported the use of combined operative placement of pulmonary artery bands with catheter-placed ductal stents [5]. Atrial septal defects were created either surgically or via balloon intervention in the cardiac catheterization lab. This combined approach satisfied the goals for first-stage palliation. In the current setting, a "hybrid" approach can consist of many variations of transvascular procedures and banding the pulmonary arteries as a primary intervention $[6 \bullet \bullet]$.

\section{Goals of Initial Palliation of Hypoplastic Left Heart Syndrome}

Regardless of the procedure performed, Norwood procedure, Sano modification of the Norwood procedure, or hybrid palliation, the goals of first-stage palliation for HLHS remain the same (see Table 1): unobstructed systemic cardiac output; adequate, but not excessive, pulmonary blood flow; unimpaired coronary blood flow; and adequate mixing of systemic and pulmonary venous return with no obstruction of pulmonary venous return.

In addition to these goals, it should also be noted that unobstructed systemic venous return is also necessary, but does not usually need to be addressed surgically in these patients.

The means used to achieve these goals differs depending on the procedure used for initial palliation (see Table 1). For the Norwood procedure and the Sano modification of the Norwood procedure, unobstructed systemic blood flow and unimpaired coronary blood flow are achieved with a Damus-Kaye-Stansel (DKS) anastomosis in combination with an aortic arch reconstruction. In the hybrid procedure, systemic blood flow is achieved via the stenting of the ductus arteriosus (see Fig. 1), or by maintaining ductal patency using prostaglandin E1. In some cases, the use of a reverse, modified Blalock-Taussig shunt has been suggested to provide adequate retrograde flow to the coronary arteries $[7,8]$.

Each type of palliation uses a different approach to provide adequate, but not excessive, pulmonary blood flow. In the

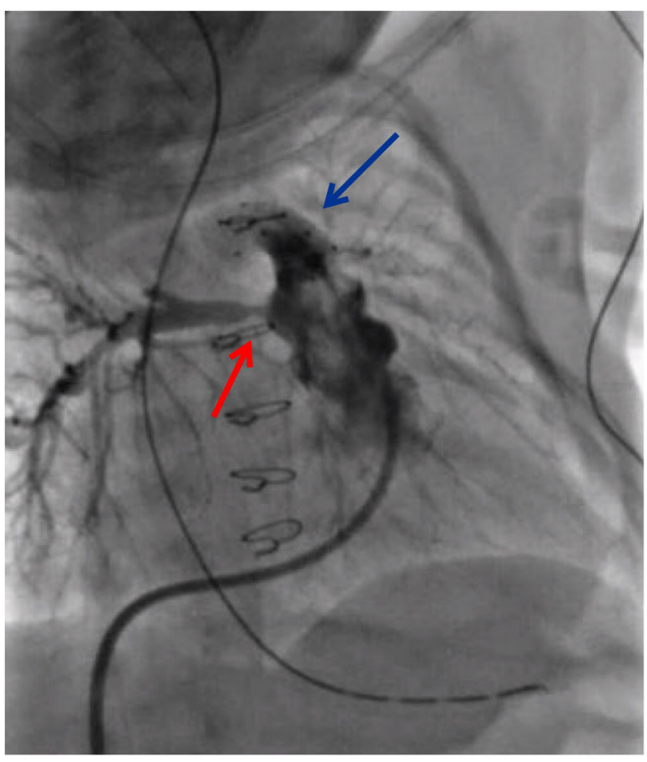

Fig. 1 Radiographic image showing pulmonary artery band (red arrow) and ductus arteriosus stent (blue arrow)

Norwood procedure, a modified Blalock-Taussig shunt is placed between the innominate artery and the pulmonary artery, usually on the right side. In the Sano modification, a conduit is placed between the right ventricle and the pulmonary artery. The Sano conduit is classically a valveless conduit, although some centers report good outcomes with a valved femoral vein homograft [9]. The main reported benefit of the latter homograft is improved growth of the pulmonary arteries.

For the stage 1 hybrid procedure, since the main pulmonary artery remains in continuity with the branch pulmonary arteries, the challenge is appropriately decreasing the amount of pulmonary blood flow rather than providing a separate stable source of pulmonary blood. To this end, pulmonary artery banding is employed. Since the main pulmonary artery is the only outlet for cardiac output to both the systemic and pulmonary circulations, pulmonary artery bands are placed more distally on the right and left pulmonary arteries, rather than on the main pulmonary artery (see Fig. 1). In this figure, the red (lower) arrow identifies the pulmonary artery band that is located on the right pulmonary artery. The blue (upper) arrow identifies the stent in the ductus arteriosus.

Table 1 Anatomic and physiologic goals of stage 1 palliation of hypoplastic left heart syndrome

\begin{tabular}{ll}
\hline Stage 1 goals & Norwood \\
\hline $\begin{array}{l}\text { 1. Unobstructed systemic circulation with } \\
\text { unimpaired coronary blood flow }\end{array}$ & $\begin{array}{c}\text { Damus-Kaye-Stansel procedure } \\
\text { with aortic arch reconstruction } \\
\text { Modified Blalock-Taussig shunt or } \\
\text { RV to PA conduit } \\
\begin{array}{l}\text { 2. Controlled source of pulmonary blood flow } \\
\text { into the right atrium }\end{array}\end{array}$ \\
\hline
\end{tabular}




\section{Complications of the Hybrid Procedure}

While none of these approaches is without complication, there are some complications that are specific to the hybrid approach. In some studies, the rate of reintervention is increased in patients undergoing the hybrid procedure [10], whereas in others, this has not been the case [11]. This variation in re-intervention may be explained by differences in the indications for the hybrid procedure, as some centers only use hybrid palliation for high-risk patients with HLHS. The reasons for reintervention in patients undergoing hybrid palliation of HLHS include:

1. Obstruction to retrograde flow in the aortic arch

2. Increased gradient across the atrial septal defect

3. Stenosis of the ductal stent due to intimal proliferation

4. Re-coarctation distal to the ductal stent

5. Pulmonary artery stenosis, especially of the left pulmonary artery

Many re-interventions can be addressed in the cardiac catheterization laboratory, although some may require an open operative procedure. In some cases, conversion to a Norwood or a Sano modification may be necessary. Since many of these re-interventions require anesthesia, consideration needs to be given to the possible effects of repeated exposure to anesthetics on neurodevelopmental outcomes. Although many studies have examined the neurodevelopmental effects of anesthetics in young children, few have investigated their effects in children with single-ventricle physiology. Fetal ultrasound and MRI have demonstrated abnormalities in cerebral blood flow, structural brain abnormalities, and white matter injury in HLHS patients. Furthermore, post-natal MRI changes consistent with hemorrhagic and thromboembolic infarction are commonly found in these patients, further obfuscating the etiology of any subsequent neurocognitive deficits $[12 \bullet \cdot$. In one recent study, a greater cumulative exposure to volatile anesthetics was associated with worse neurodevelopmental outcomes in patients with HLHS [13•]. Additional research in this area is required before any consensus can be reached about the neurodevelopmental effects of anesthetic agents in these fragile infants.

Concern has also been raised that the components of the hybrid procedure may render the comprehensive stage 2 operation more difficult. Aortic arch reconstruction may be technically more challenging in the presence of the ductal stent, and pulmonary artery stenosis might present a reconstructive challenge or lead to non-operative re-interventions as discussed above $[14,15]$.

\section{Anesthetic Considerations After Stage 1 Palliation of Hypoplastic Left Heart Syndrome}

In many instances, infants who have had a stage 1 palliative procedure for HLHS are cared for by subspecialty pediatric cardiac anesthesia teams. In many centers, pediatric cardiac anesthesiologists render care to all children with significant congenital heart disease. However, this is not always the case, and general pediatric anesthesiologists may have to provide care for these patients. These infants can present for a variety of procedures before their stage 2 procedure. A number of procedures including gastrostomy feeding tube and semipermanent venous access placement, MRI scans, and airway procedures will require anesthesia or sedation to be completed. In addition, repeated trips to the cardiac catheterization lab may be required. Some centers now use cardiac MRI in lieu of catheterization for preoperative planning, so anesthesia or sedation will be needed for these procedures as well [16••].

In general, successful anesthesia care for these infants requires meticulous attention to intravascular volume, maintenance of adequate, but not excessive, systemic vascular resistance (SVR), and balancing the systemic and pulmonary vascular resistance (PVR). Excessive oxygen delivery and hyperventilation can reduce the pulmonary vascular resistance, increasing pulmonary blood flow to dangerous levels. Consideration should be given to withholding angiotensinconverting enzyme inhibitors on the morning of surgery as the combination of these medications with many commonly used anesthetic agents has resulted in severe hypotension on induction that might initially be difficult to manage [17]. Although invasive blood pressure monitoring might seem to be desirable in patients with HLHS undergoing non-cardiac procedures, there are some reasons to avoid it. First, arterial access may be technically difficult to achieve. Second, attempting arterial access may make it difficult to achieve said access in future major procedures. Third, the time to achieve successful access may unnecessarily prolong the anesthetic. Most brief procedures without anticipated major fluid shifts can be undertaken in these infants without invasive monitoring. Other monitoring techniques such as cerebral and somatic oximetry may be utilized to assess organ perfusion [18]. If central venous access is already in place, venous oxygen saturation and lactate levels can help guide care. If central venous access is not present, the same considerations of time and risk of placement should be considered as they may outweigh any benefits. Anticipating the need for repeat cardiac surgery, and that the superior vena cava will be used to complete the second stage, care must be taken to avoid injury to or thrombosis of the vena cava.

For most brief procedures, the airway should be extubated immediately after the procedure. Exceptions would include those infants who are already intubated before the procedure 
or who are undergoing airway procedures that preclude extubation. Postoperative tracheal intubation and ventilator support should also be considered if the infant develops any respiratory or hemodynamic instability during the procedure.

Recently, many question the safety of performing laparoscopic procedures in these infants. Historically, singleventricle physiology was considered a contraindication for laparoscopic procedures, although this has not been the case of late. A majority of gastrostomy feeding tubes at our institution are now placed using laparoscopic techniques. Low inflating pressures along with close communication between the surgeon and the anesthesiologist can ensure good outcomes for these infants. Nonetheless, there should be a low threshold for conversion to an open procedure.

The considerations for general anesthesia in these infants are similar for all types of stage 1 palliation, although some differences warrant mention. Infants with a classic Norwood procedure and a modified BlalockTaussig shunt are at increased risk of becoming unstable if the PVR is reduced substantively. The reduced PVR will tip the balance toward excessive pulmonary blood flow with subsequently decreased systemic blood flow. In addition, diastolic runoff into the pulmonary circulation will reduce the diastolic blood pressure, which could decrease coronary perfusion pressure, resulting in inadequate myocardial blood flow. Poor perfusion of the myocardium can decrease cardiac output and increase the frequency of arrhythmias, either of which can be rapidly fatal. These patients require meticulous balancing of the systemic and pulmonary vascular resistances to ensure adequate organ homeostasis. Prolonged fasting periods are not recommended in these infants as the combination of polycythemia and hypovolemia can increase the risk for shunt thrombosis and occlusion, which constitutes a life-threatening emergency. Adequate hydration either orally or via a gastrostomy tube will decrease the risk of this occurrence. Preoperatively, if hydration cannot be maintained by enteral means, then intravenous access should be established to deliver fluids. All diuretics should be withheld on the morning of the procedure; serum electrolyte concentrations should be evaluated preoperatively.

Infants with the Sano modification of the Norwood procedure require less meticulous balancing of the systemic and pulmonary vascular resistances, although due consideration remains prudent. Excessive decreases in the systemic vascular resistance may decrease pulmonary blood and increase the severity of cyanosis, which may not be well tolerated. Complications specific to the Sano modification are proximal shunt obstruction at the ventricular level and progressive diffuse shunt obstruction [4]. Proximal shunt obstruction may be episodic in nature and can resemble a "Tet" spell. In fact, beta blockers may be employed to decrease the severity of the shunt obstruction. Progressive diffuse shunt obstruction is detected by echocardiography and oxygen saturation levels. If the oxygen saturation is less than anticipated, the cause should be investigated. This may require a visit to the catheterization lab. Non-cardiac procedures would be unadvisable in this case until the cause of the decreased oxygen saturation is ascertained.

For infants with hybrid procedures including pulmonary artery banding, the PVR becomes much less of a consideration, but careful control of the SVR remains critical. If the SVR is allowed to decrease significantly, blood flow to the cerebral and coronary arteries could be compromised. Obstruction to retrograde flow in the aortic arch may also occur in these patients. Accordingly, evidence of obstruction should be sought via a preoperative echocardiogram. Increased tricuspid regurgitation or worsening right ventricular function can be early signs of obstruction [19]. Although detecting obstruction to blood flow in the retrograde aortic arch would be difficult intraoperatively, cerebral oximetry might detect a decrease in cerebral blood flow that could indicate obstruction. Close attention to the EKG may allow early detection of decreased coronary blood flow. Prolonged fasting times may not be well tolerated and should be avoided; maintenance of adequate, but not excessive, intravascular volume will decrease the likelihood of a thrombotic event in the ductal stent.

Induction of anesthesia may be accomplished using any of the commonly available induction agents. An inhalation induction is a reasonable choice if an intravenous line is not already in place. A smooth inhalation induction can attenuate the stress response and may also facilitate line placement. Alternately, an intravenous induction of anesthesia may be selected if intravenous access is present. However, if intravenous access is not present, restraining an unsedated, crying infant for multiple attempts at intravenous cannulation may lead to adverse hemodynamic consequences. Certainly, it is not unreasonable to limit the cannulation attempts to one or two by an individual experienced in this area. In terms of induction agents, propofol, ketamine, and etomidate are all excellent choices. If propofol is selected, it is preferable to titrate smaller doses to prevent large swings in circulatory indices. Alternately, ketamine may be used as the sole induction agent or as an adjunct to other anesthetics and analgesics. In the doses commonly used to induce anesthesia, its effect on the PVR will be minimal and it will maintain the SVR. Etomidate may also be used for induction of anesthesia. It provides a smooth induction with minimal hemodynamic fluctuations. If tracheal extubation is not anticipated at the conclusion of surgery, a high-dose opioid induction technique may also be considered. When extubation is planned, the anesthetic agents should be 
chosen carefully; agents such as dexmedetomidine and remifentanil can be considered. These medications attenuate the stress response of the procedure, and should facilitate extubation at the end of the procedure. Although many of the procedures that are performed do not result in substantive postoperative pain, for those that do, a postoperative pain strategy needs to be in place. Fentanyl (1 to 2 micrograms per kilogram intravenously) is often all that is needed, but one should anticipate that some of these infants may have developed tolerance to opioids and sedatives, especially after a prolonged stay in the intensive care unit. Many different muscle relaxants may also have been administered to these infants. There are no specific recommendations for the choice of a non-depolarizing muscle relaxant. Usage is usually determined by the duration of the procedure and the preference of the anesthesiologist. If a relaxant has been used, then a neuromuscular blockade monitor should track the twitch responses throughout. The choice of an anticholinesterase or sugammadex will be determined by the twitch responses, the availability of the drugs, and the preference of the anesthesiologist.

\section{Conclusions}

Although much progress has been made in the last 40 years, the future of care for infants who are born with HLHS still remains unclear. When hybrid palliation first gained popularity, it was thought that this palliative approach would improve survival and neurologic outcomes. By postponing surgery that requires cardiopulmonary bypass, and is of greater duration until the brain is more mature, it was hoped that neurodevelopmental outcomes would improve. However, at this time, the current evidence does not support improved neurodevelopmental outcomes when the hybrid procedure for stage 1 palliation of HLHS is used [20,21]. Additionally, it was thought that the hybrid technique would reduce overall risk and increase survival when used as an alternative to the Norwood or the Sano modification of the Norwood, but this has been difficult to prove [21, 22]. Even though some centers use the hybrid as stage 1 palliation for all infants with HLHS, many centers have reserved its use for those infants at greatest risk for Norwood procedures. The use of the hybrid technique for only the highest risk patients has made it difficult to compare outcomes among the different stage 1 strategies. A very recent metaanalysis suggested that early survival is decreased in patients undergoing hybrid stage 1, although no definite conclusion could be made at this time [23••]. For those infants at high risk for the Norwood procedure, the hybrid alternative can provide good midterm results. However, results in infants with intact atrial septum and aortic atresia remain disappointing [24]. On the other hand, infants with risk factors such as prematurity and low birth weight may benefit from the hybrid approach. In one study, the Norwood operation with a Sano modification provided the best overall survival, although the hybrid techniques may have improved survival in infants with low birth weight [25••]. For now, the stage 1 approach to HLHS remains largely center dependent. Whether this will change in the future remains to be determined.

Some have suggested that the definition of successful outcome for these complex infants may need to be revised. Classically, success was defined as freedom from death or transplantation. The future of transplantation is changing rapidly as well. Neonatal transplantation leads to very good survival and generally high quality of life, but neonatal donor hearts are not always available when needed. This has led to a renewed interest in xenotransplantation in neonates [26•]. What the future will bring in this area remains unknown, but as anesthesiologists we may need to be prepared to care for these patients in the future. If transplantation can improve outcomes, is it fair to consider transplantation a failure [27]?

As it seems likely that various approaches to stage 1 palliation will continue to be used, the pediatric anesthesiologist must familiarize him- or herself with these various techniques. While most children with HLHS are cared for in specialty centers, there is always the possibility that immediate care and stabilization may need to be undertaken in the absence of an experienced pediatric cardiac anesthesiologist. There is a paucity of data in the current literature regarding the anesthetic care of these infants. Naguib et al. have published a comprehensive paper on the care of these infants while they are undergoing the hybrid stage 1 palliation. It is their conclusion that these patients have relatively stable intraoperative and postoperative hemodynamics, leading to a more straightforward anesthetic [28]. One hopes that this relative stability would make any anesthetics required before the next cardiac surgery less complicated as well.

For now, it can be said that this patient population will continue to provide challenges for the pediatric anesthesiologist. Although the future remains unclear, the hope is that with continued research and dedication to the care of these complex patients, the long-term outcomes and quality of life will steadily improve.

\section{Compliance with Ethical Standards}

Conflict of Interest Dawn M. Sweeney and Jane Arcadi declare they have no conflict of interest.

Human and Animal Rights and Informed Consent Neither Dr. Arcadi nor Dr. Sweeney performed any animal or human subject studies in this article. 


\section{References}

Papers of particular interest, published recently, have been highlighted as:

- Of importance

•- Of major importance

1. Norwood WI, Kirklin JK, Sanders SP. Hypoplastic left heart syndrome: experience with palliative surgery. Am J Cardiol. 1980;45(1):87-91.

2. Bailey LL, Nehlsen-Cannarella SL, Concepcion W, Jolley WB. Baboon-to-human cardiac xenotransplantation in a neonate. JAMA. 1985;254(23):3321-9.

3. Norwood WI, Lang P, Hansen DD. Physiologic repair of aortic atresia-hypoplastic left heart syndrome. N Engl J Med. 1983;308(1):23-6.

4. Sano S, Ishino K, Kawada M, Arai S, Kasahara S, Asai T, et al. Right ventricle-pulmonary artery shunt in first-stage palliation of hypoplastic left heart syndrome. J Thorac Cardiovasc Surg. 2003;126(2):504-9.

5. Gibbs JL, Wren C, Watterson KG, Hunter S, Hamilton JR. Stenting of the arterial duct combined with banding of the pulmonary arteries and atrial septectomy or septostomy: a new approach to palliation for the hypoplastic left heart syndrome. Br Heart J. 1993;69(6): $551-5$.

$6 . \bullet$ Haller C, Caldarone CA. The evolution of therapeutic strategies: niche apportionment for hybrid palliation. Ann Thorac Surg. 2018;106(6):1873-80. https://doi.org/10.1016/j.athoracsur.2018. 05.048 Comprehensive yet very readable article outlining the potential, uses, and variations of the hybrid procedure.

7. Baba K, Honjo O, Chaturvedi R, Lee KJ, Van Arsdell G, Caldarone CA, et al. "Reverse Blalock-Taussig shunt": application in single ventricle hybrid palliation. J Thorac Cardiovasc Surg. 2013;146(2): 352-7. https://doi.org/10.1016/j.jtcvs.2012.11.029. Epub 2012 Dec 8.

8. Caldarone CA, Benson LN, Holtby H, Van Arsdell GS. Main pulmonary artery to innominate artery shunt during hybrid palliation of hypoplastic left heart syndrome. J Thorac Cardiovasc Surg. 2005;130(4):e1-2.

9. Briceno-Medina M, Kumar TKS, Sathanandam S, Boston U, Perez M, Allen J, et al. Femoral vein homograft as Sano shunt results in improved pulmonary artery growth after Norwood operation. Cardiol Young. 2018;28(1):118-25. https://doi.org/10.1017/ S1047951117001688.

10. Nwankwo UT, Morell EM, Trucco SM, Morell VO, Kreutzer J. Hybrid strategy for neonates with ductal-dependent systemic circulation at high risk for Norwood. Ann Thorac Surg. 2018;106(2): 595-601. https://doi.org/10.1016/j.athoracsur.2018.03.007.

11. Brescia AA, Jureidini S, Danon S, Armbrecht E, Fiore AC, Huddleston CB. Hybrid versus Norwood procedure for hypoplastic left heart syndrome: contemporary series from a single center. J Thorac Cardiovasc Surg. 2014;147(6):1777-82. https://doi.org/10. 1016/j.jtcvs.2014.02.066.

12.• Lloyd DF, Rutherford MA, Simpson JM, Razavi R. The neurodevelopmental implications of hypoplastic left heart syndrome in the fetus. Cardiol Young. 2017;27(2):217-23. https:// doi.org/10.1017/S1047951116001645 This is the only article I have seen looking at fetal neurodevelopment in hypoplastic left heart syndrome. There is a good discussion of abnormalities of cerebral blood flow in fetuses with HLHS.

13. Diaz LK, Gaynor JW, Koh SJ, Ittenbach RF, Gerdes M, Bernbaum $\mathrm{JC}$, et al. Increasing cumulative exposure to volatile anesthetic agents is associated with poorer neurodevelopmental outcomes in children with hypoplastic left heart syndrome. J Thorac Cardiovasc
Surg. 2016;152(2):482-9. https://doi.org/10.1016/j.jtcvs.2016.03. 095 This is the only paper that looks at cumulative exposure to volatile anesthetics in HLHS specifically. While there are no definitive answers on the subject, anesthesiologists need to remain vigilant in the care of these infants who may be at risk for poorer neurodevelopmental outcomes.

14. Yerebakan C, Valeske K, Elmontaser H, Yörüker U, Mueller M, Thul J, Mann V, Latus H, Villanueva A, Hofmann K, Schranz D, Akintuerk H. Hybrid therapy for hypoplastic left heart syndrome: myth, alternative, or standard? J Thorac Cardiovasc Surg. 2016;151(4):1112-21, 1123.e1-5. https://doi.org/10.1016/j.jtcvs. 2015.10.066. This article discusses in detail the reinterventions commonly needed after hybrid palliation. The discussion section provides further insight into this issue.

15. Mosca RS. Hybrid therapy for hypoplastic left heart syndrome: myth, alternative, or standard - neither minotaur nor Midas. J Thorac Cardiovasc Surg. 2016;151(4):1123-5. https://doi.org/10. 1016/jtcvs.2015.10.099 Epub 2015Oct 30.

16.• Ohye RG, Schranz D, D'Udekem Y. Current therapy for hypoplastic left heart syndrome and related single ventricle lesions. Circulation. 2016;134(17):1265-79 Excellent review of therapy for HLHS, including all 3 stages of palliation.

17. Watkins SC, McNew BS, Donahue BS. Risks of noncardiac operations and other procedures in children with complex congenital heart disease. Ann Thorac Surg. 2013;95:2014-1.

18. Mittnacht AJC. Near infrared spectroscopy in children at high risk of low perfusion. Curr Opin Anaesthesiol. 2010;23:342-7.

19. Galantowicz M, Cheatham JP, Phillips A, Cua CL, Hoffman TM, Hill SL, et al. Hybrid approach for hypoplastic left heart syndrome: intermediate results after the learning curve. Ann Thorac Surg. 2008;85(6):2063-70. https://doi.org/10.1016/j.athoracsur.2008.02. 009 .

20. Cheatham SL, Carey H, Chisolm JL, Heathcock JC, Steward D. Early results of neurodevelopment following hybrid stage I for hypoplastic left heart syndrome. Pediatr Cardiol. 2015;36(3):685-91. https://doi.org/10.1007/s00246-014-1065-5.

21. Knirsch W, Liamlahi R, Hug MI, Hoop R, von Rhein M, Prêtre R, et al. Mortality and neurodevelopmental outcome at 1 year of age comparing hybrid and Norwood procedures. Eur J Cardiothorac Surg. 2012;42(1):33-9. https://doi.org/10.1093/ejcts/ezr286.

22. Malik S, Bird TM, Jaquiss RD, Morrow WR, Robbins JM. Comparison of in-hospital and longer-term outcomes of hybrid and Norwood stage 1 palliation of hypoplastic left heart syndrome. J Thorac Cardiovasc Surg. 2015;150(3):474-80.e2. https://doi.org/ 10.1016/j.jtcvs.2015.06.071.

23.• Cao JY, Lee SY, Phan K, Ayer J, Celermajer DS, Winlaw DS. Early outcomes of hypoplastic left heart syndrome infants: meta-analysis of studies comparing the hybrid and Norwood procedures. World J Pediatr Congenit Heart Surg. 2018;9(2):224-33. https://doi.org/10. $1177 / 2150135117752896$ This is the only meta-analysis specifically comparing the Norwood procedure with the hybrid procedure.

24. Murphy MO, Bellsham-Revell H, Morgan GJ, Krasemann T, Rosenthal E, Qureshi SA, et al. Hybrid procedure for neonates with hypoplastic left heart syndrome at high- risk for Norwood: midterm outcomes. Ann Thorac Surg. 2015;100(6):2286-90; Discussion 2291-2. https://doi.org/10.1016/j.athoracsur.2015.06.098.

25.• TJ W, McCrindle BW, Hickey EJ, Ziemer G, Tchervenkov CI, Jacobs ML, et al. Is a hybrid strategy a lower-risk alternative to stage 1 Norwood operation? J Thorac Cardiovasc Surg. 2017;153(1):163-172.e6. https://doi.org/10.1016/j.jtcvs.2016.08. 021 This article compares both conventional Norwood procedures and those with the RV to PA conduit modification to the hybrid procedure. Most papers do not compare all 3 types of palliation. 
26. Cleveland D, Adam Banks C, Hara H, Carlo WF, Mauchley DC, DKC C. The case for cardiac xenotransplantation in neonates: is now the time to reconsider xenotransplantation for hypoplastic left heart syndrome? Pediatr Cardiol. 2019;40(2):437-44. https://doi. org/10.1007/s00246-018-1998-1 Interesting article about a possible future of cardiac transplantation in neonates that discusses genetic engineering and xenotransplantation.

27. Wilder TJ. Redefining successful outcomes in the hybrid era. World J Pediatr Congenit.
28. Naguib AN, Winch P, Schwartz L, Isaacs J, Rodeman R, Cheatham JP, et al. Anesthetic management of the hybrid stage 1 procedure for hypoplastic left heart syndrome (HLHS). Paediatr Anaesth. 2010;20(1):38-46. https://doi.org/10.1111/j.1460-9592.2009. 03205.x.

Publisher's Note Springer Nature remains neutral with regard to jurisdictional claims in published maps and institutional affiliations. 